\title{
O PAPEL DA ACADEMIA BRASILEIRA DE NEUROLOGIA NO ENSINO DA NEUROLOGIA
}

\author{
UMA VISÃO PESSOAL
}

MARCO AURÉLIO LANA-PEIXOTO *

\begin{abstract}
RESUMO - A Academia Brasileira de Neurologia (ABN) tem demonstrado crescente interesse pelo ensino da neurologia, tanto na graduação quanto na residência médica. Ela deve definir o padrão da prática da neurologia no país, traçar o perfil de competência mínima do neurologista, determinar a competência mínima em neurologia do médico não-neurologista, desenvolver suas próprias atividades de ensino, e definir e propiciar o desenvolvimento das qualidades humanísticas desejáveis no neurologista. A ABN deve formular um currículo mínimo de neurologia na graduação e na residência médica e esforçar por sua adoção pelas escolas médicas e pelos Programas de Residência. Além disso, a ABN deve, por si própria, tornar-se agente efetora do ensino através: de um Programa de Educação Continuada, promovendo cursos e simpósios; da elaboração de um sistema de ensino programado em neurologia, via postal; da implantação de videoteca e de banco de dados da literatura. A Comissão de Ensino deve desempenhar papel fundamental em todas as atividades de ensino promovida pela $\mathrm{ABN}$, definindo sua política, estabelecendo prioridades e coordenando as ações de ensino dos Grupos de Trabalho e Pesquisa.
\end{abstract}

The role of the Brazilian Academy of Neurology in the teaching of neurology: a personal view

SUMMARY - The Brazilian Academy of Neurology (ABN) may play an important role for a better education of medical students and residents in neurology in Brazil. Its field of action ranges from suggesting a core curriculum in neurology to Medical Schools and Residency Programs to assess the quality of teaching they offer in order to guide students and graduates who apply for positions. In addition to that ABN may be itself a teaching institution offering educational activities in a Program of Continuing Medical Education with a well-designed system of credits. Courses on basic sciences, methods of diagnosis and therapeutic advances, symposia and workshops as well as a Programmed Teaching in Neurology suitable to residents and pnactioners are to be included. A video collection and a bank of diata on the current literature may widen the available! set of services. The ABN Commitee on Teaching is to play a central role defining policy, deciding on priorities, planning and establishing timing and placing of all educational (activities. Of utmost importance is to define humanistic qualities desirable in the neurologist identifying qualities students and residents bring to the educational process and those acquired in the practice of neurology. The cognitive dimensions of moral and ethics can be taught and should be regularly included in the educational activities sponsored by the Academy.

A Academia Brasileira de Neurologia (ABN) tem demonstrado interesse crescente pelas questões do ensino de neurologia no país, tanto no curso de graduação quanto na residência médica. A Comissão de Ensino (CE) da ABN, em sucessivos biênios, tem discutido e publicado em seus Relatórios as linhas gerais dos objetivos e da temática deste ensino 1.2,8,10. Cursos, palestras e mesas-redondas sobre o ensino têm sido freqüentemente incluidos nos programas dos Congressos Brasileiros de Neurologia.

* Professor Adjunto de Neurologia, Faculdade de Medicina, Universidade Federal de Minas Gerais.

Dr. Marco A. Lana-Peixoto - Departamento de Neurologia, Instituto Hílton Rocha - Av. Anel da Serra 13S5 - 80210 Belo Horizonte MG - Brasil. 
A nítida preocupação da $A B N$ com o ensino da neurologia parece demonstrar que ela tem consciência de portar alguma responsabilidade tanto na definição do paradigma da neurologia brasileira, quanto na determinação dos caminhos para atingir o padrão desejado. No entanto, nas atividades da ABN e entre nós mesmos, seus membros, pairam dúvida e contradições em relação a esta responsabilidade. Por que seria da ABN e de nós próprios neurologistas, e não do Estado ou de outros segmentos da sociedade, o dever de traçar o perfil desejável da prática neurológica em nosso meio? O que torna a $\mathrm{ABN}$, e portanto nós mesmos, moralmente responsáveis pela qualidade da neurologia que se pratica em todo este país? Estas questões de natureza moral se colocam acima de quaisquer preceitos legais. A ABN é uma sociedade civil, autônoma e independente do Estado, não tendo entre suas obrigações legais qualquer dever junto aos médicos em relação à sua educação e qualificação profissional, nem junto à sociedade em relação à determinação de parâmetros de qualidade da assistência médica prestada. Portanto, em termos legais, a ABN nada deve aos médicos em relação à sua educação e nada deve à sociedade em relação à sua proteção, pelo estabelecimento de critérios de competência ou definição de padrões de conduta. No entanto, o que faz a ABN moralmente responsável, em relação à educação médica e em relação à sociedade, é a própria consciência de suas possibilidades e competências. Em seu Estatuto, entre suas finalidade, ela se propõe «estimular e patrocinar a formação de novos profissionais em Neurologia e ciências afins»e «promover e patrocinar o aprimoramento de seus associados...». A busca ordenada do cumprimento destas suas finalidades educacionais pode modificar o perfil da neurologia brasileira. É o conhecimento desta possibilidade que traz em si o dever moral de se tornar agente transformadora da neurologia praticada neste país.

A ABN deve portanto: (1) definir o padrão da prática neurológica no país; (2) traçar o perfil de competência do neurologista; (3) avaliar a necessidade de neurologistas para as várias regiões do país; (4) planejar a formação dos especialistas conforme as necessidades regionais; (5) determinar a competência mínima em neurologia do médico não-neurologista; (6) desenvolver suas próprias atividades de ensino; (7) propiciar o desenvolvimento das qualidades humanistas dos neurologistas.

\section{A ABN E O ENSINO NA GRADUAÇÃO}

Para melhorar a prática neurológica em nosso meio é necessário que a $\mathrm{ABN}$ atue inicialmente junto aos cursos de graduação nas escolas médicas do país. Ela deve sugerir o que ensinar, estruturando a composição de um currículo mínimo em neurologia que contenha desde as neurociências básicas, a propedêutica clínica e laboratorial, a neuropatologia, até a clínica neurológica centralizada no cuidado individual do paciente. Este modelo curricular deve ser analisado em todas as suas partes, com explicação e justificativa de cada ponto de seu conteúdo.

A partir de sua elaboração, o próximo passo seria a adoção deste modelo pélas escolas médicas. Como a ABN congrega, entre seus membros, docentes da maioria das escolas médicas do país, caberia a este corpo docente a luta, em linha de frente, para que este currículo fosse implantado em suas respectivas escolas. Além disto, a $\mathrm{ABN}$ deveria publicar sua proposta curricular em revista de educação médica, assim como encaminhá-la a todos os professores de neurologia e aos diretores de Faculdades de Medicina do país. A adequada divulgação do conteúdo programático de seu currículo, com suas justificativas e mesmo estratégias de implantação, é indispensável para sua concretização.

Uma vez adotado, o currículo deverá receber avaliação crítica periódica por exame de amostragem de alunos, promovida pela $\mathrm{CE}$ da $\mathrm{ABN}$. Apenas mediante estas avaliações periódicas a validade do modelo curricular proposto pode ser testada e possíveis lacuna» corrigidas.

\section{A ABN E O ENSINO NA RESIDÊNCIA MÉDICA}

A residência médica é a mais adequada das formas pana a formação do neurologista 7 . JÊ durante esse período que o médico adquire hábitos e atitudes, assim como desenvolve habilidades e competências que o transformam em um neurologista.

A ABN pode atuar eficazmente nos Programas de Residência Médica em Neurologia em todo o país, promovendo sua melhor qualificação e integração. Inicialmente, há necessidade de formulação de um «currículo mínimo» capaz de propiciar capacitação técnica e desenvolvimento humanístico, indispensáveis para a formação do neurologista. Os programas de Residência em Neurologia» oferecidos pelas várias instituições do país, serão então credencia- 
dos pelia $\mathrm{ABN}$, quando preencherem os requisitos dispostos no «currículo mínimo» proposto. A CE da ABN deve avaliar periodicamente estes Programas de Residência em Neurologia, por ela credenciados, assim como categorizá-los segundo sua qualidade. Esta categorização será útil em dois aspectos. Primeiro, servirá como guia de orientação aos médicos que desejarem ingressar em algum Programa de Residência. Segundo, estimulará, por sadia competitividade, o aprimoramento didático e o crescimento das iniciativas de ensino e de investigação clinica das várias residências no país.

A ABN poderá, também, fomentar o intercâmbio entre as residências de neurologia para estágios de médicos residentes e docentes em áreas específicas da especialidade em instituições que ofereçam melhores condições de aprendizado. Este intercâmbio funcionará como meio propulsor do desenvolvimento da neurologia em todas as regiões do Brasil, fazendo com que especialistas bem treinados, ao regressarem para suas localidades de origem, promovam a formação de núcleos de estudo e de treinamento nessas áreas específicas. Igualmente, a nível internacional, a ABN também poderá agir, por intercâmbio para «fellowahip» em instituições do exterior.

\section{A ABN COMO EFETORA DO ENSINO}

Além de sugerir currículos mínimos de neurologia para a graduação médica e para oa Programas de Residências em Neurologia, de criar um sistema de avaliação periódica desses currículos e das instituições, de ensino, de credenciar os Programas de Residência e de categorizá-los periodicamente, e de promover o intercâmbio nacional e internacional para a melhor formação e qualificação de residentes e docentes, a $A B N$ deve, por si própria tornar-se «agente efetora» do ensino da neurologia. As atividades de ensino propiamente dito, promovidas pela $\mathrm{ABN}$, deveriam centralizar-se na promoção e na efetivação de: (1) Programa de Educação Continuada em Neurologia; (2) Sistema de Ensino Programado em Neurologia; (3) Videoteca; (4) Biblioteca ou Banco de Dados e Referências.

O «Programa de Educação Continuada em Neurologia» deve constituir-se na principal atividade de ensino da $\mathrm{ABN}$. Ele deve ser planejado de forma racional e lógica, com cursos periódicos de disciplinas básicas, de métodos de diagnóstico laboratorial e por imagem, de terapêutica e de atualização clínica. Cursos de metodologia científica, de análise estatística, de leitura e redação de trabalhos científicos devem também ser incluídos.

A efetivação do «Sistema de Ensino Programado» pode representar importante passo educacional da ABN. Este projeto consiste num sistema de leitura orientada dos mais significativos trabalhos publicados nas várias áreas da neurologia. Cópias destes artigos, selecionados pelos Grupos de Trabalho ou pela CE, seriam encaminhados, via postal, junto a uma análise e orientação de sua leitura, a todos os inscritos no Programa. Este sistema se compõe de duas modalidades: (1) Ensino Fundamental Programado; e (2) Atualização Programada. O «Ensino Fundamental Programado» seria dirigido aos médicos residentes e a todos aqueles que desejarem obter núcleo sólido de informação nas várias áreas da especialidade. A «Atualização Programada» consistiria na divulgação anual de análise e apreciação crítica dos mais importantes trabalhos publicados na literatura, naquele ano. Seria um «Year Book» mais diretivo, mais crítico e voltado aos interesses e patamares da neurologia brasileira. Também editada pelos vários Grupos de Trabalho, a Atualização Programada seria enviada junto às cópias dos artigos a todos os inscritos. O Sistema de Ensino Programado favoreceria, portanto, todos os neurologistas brasileiros, principalmente aqueles que, devido às dificuldades econômicas e geográficas, não têm acesso às maiores bibliotecas ou às promoções de ensino mais convencionais da $\mathrm{ABN}$.

A «Videoteca» é recurso de grande alcance educacional, capaz de levar os cursos, simpósios e congressos programados pela $\mathrm{ABN}$ e por congêneres de outros países a todos os neurologistas. A ABN deverá manter sua videoteca sempre atualizada, adquirindo vídeos de eventos científicos nacionais e internacionais, anunciando periodicamente suas aquisições e facilitando a divulgação desses vídeos.

A «Biblioteca» da ABN seria melhor chamada de «Banco de Dados e Referências». A ABN poderia fornecer, quando requisitada, levantamento de dados científicos e de referências bibliográficas obtidas por programas de computação. Estes programas, já disponíveis n,os Estados Unidos, para uso individual e de bibliotecas, funcionam em forma de assinaturas com atualização permanente de toda a literatura neurológica.

Estas formas diretas de prestação de serviço educacional e científico pela ABN seriam atrativos importantes a todos os neurologistas que manteriam, então, contato mais próximo e frequente com a instituição, ao se sentirem, portanto, beneficiados por ela. 


\section{O ENSINO PELA ABN: ESTRATÉGIAS E TÁTICAS}

A fim de que a ABN possa efetuar com sucesso suas atividades de ensino, planos e estratégias de ação se tornam necessários. Em primeiro lugar, cabe à CE desempenhar papel fundamental como coordenadora de todas as atividades de ensino promovidas pela ABN. A CE deve estabelecer planejamento a curto e médio prazo das atividades que serão realizadas, dentro de um calendário racional e, sempre que possível, em distribuição regional pelo país. Não podem mais as atividades didáticas e científicas da $\mathrm{ABN}$ continuar ocorrendo de forma aleatória, irregular, às vezes sobreposta numa mesma ocasião ou repetitivas no mesmo local, desconectadas entre si, arredias de um planejamento adequado.

A coordenação centnalizada dessas atividades não significa que a CE chama a si a responsabilidade de sua promoção e execução. Ao contrário, à CE competem essencialmente a formulação da política de ensino da $\mathrm{ABN}$, a definição de objetivos e a avaliação dos resultados dessa política. Igualmente a ela cabe a coordenação, pela determinação da prioridade e distribuição no tempo e espaço, das atividades científicas e didáticas da ABN. Os Grupos de Trabalho e Pesquisa deveriam tornar-se responsáveis pela promoção e execução de todas essas atividades. Eles seriam os órgãos efetores das ações de ensino, sob a coordenação da CE, em estrita colaboração com a Secretaria-Tesouraria Geral. A ABN deveria ainda, através da CE, desenvolver sua capacidade de avaliação crítica de suas ações de ensino. Por essa avaliação crítica, séria, objetiva e impessoal, desvios dos caminhos traçados podem ser corrigidos, futuros erros evitados e objetivos originalmente definidos podem ser alcançados.

A avaliação crítica periódica exercida pela CE deveria iniciar-se por suas próprias atividades, estendendo-se às dos Grupos de Trabalho e Pesquisa e atingindo até os programas educacionais dos Congressos Brasileiros de Neurologia. A relação custo-benefício das ações e eventos, o adequado aproveitamento dos recursos pessoais e das oportunidades, os resultados finais em relação as metas científicas e educacionais seriam então analisados, e sugestões corretivas então propostas. A criação de um sistema de créditos aditivos fornecidos pela ABN a neurologistas pela participação em atividades educacionais e científicas, para obtenção de «Certificados de Educação Continuada», periodicamente, poderia constituir-se também em outro atrativo das promoções da $A B N$. Estes Certificados, por outro lado, servirão para demonstrar à sociedade a permanente qualificação e a atualização científica de seus detentores. A ABN não obterá êxito na promoção do ensino enquanto ela não se fizer ouvir por todos os neurologistas do país. Isto significa que a ABN necessita de se comunicar constantemente com todos os neurologistas, informando-lhes sobre suas atividades, manifestando-lhes as resoluções de suas Comissões e órgãos Diretivos, divulgando opiniões de «experts» sobre temas controvertidos ou de grande atualidade.

Os «Arquivos de Neuro-Psiquiatria» são, desde 1970, o órgão oficial de divulgação da ABN. A revista, publicada com absoluta regularidade, indexada e presente em bibliotecas médicas de todo o mundo, tem sido pouco utilizada pela $A B N$. Especificamente em relação às ações de ensino da $\mathrm{ABN}$, as oportunidades de veiculação pelos «Arquivos» têm sido sistematicamente desperdiçadas. Por que não publicar, ainda que apenas seus sumários, os rela* tórios bienais da $\mathrm{CE}$ com suas resoluções e sugestões quanto às linhas do ensino da neurologia no país? Por que não levar ao conhecimento de toda a comunidade neurológica estas avaliações e proposições, libertando-as das amarras dos relatórios, acessíveis apenas aos membros da $\mathrm{CE}$ e aos poucos presentes às Assembléias Gerais da $\mathrm{ABN}$ ? Tivessem o trabalho $\mathbf{e}$ as sugestões da CE nestes vários anos sido publicadas, certamente teriam frutificado melhor. Igualmente, sumários de simpósios e mesas-redondas, os pontos principais apresentados em suas reuniões científicas, as conferências magnas de seus congressos, os resumos de trabalhos premiados, entre outros, merecem todos publicação pela ABN através de seu órgão oficial. Também os Grupos de Trabalho e Pesquisa deveriam se obrigar a publicar periódica e sistematicamente revisões de temas atuais em seus respectivos campos, listas dos trabalhos mais relevantes publicados na literatura internacional e apreciações críticas, entre outras. Há, portanto, necessidade de ultrapassar a ABN seus limites paroquiais e de se expressar continuamente nos «Arquivos» manifestando, a todos os neurologistas do país, sua vida e sua força.

\section{A ABN E AS DIMENSÕES HUMANISTAS DO NEUROLOGISTA}

Os grandes avanços tecnológicos de nossa era trazem em si o perigo de reduzir a medicina apenas a seus aspectos físicos, distanciando o médico das preocupações referentes à natureza humana de sua profissão. Já em 1868, Sir William Gull, refe- 
rindo-se ao malefício do dogmatismo do progresso científico, exclamava: «How often has Medicine been thus diverted from her difficult path! A discovery in physics has made us for the mament no more than galvanic batteries, or a discovery in chemistry mere oxidising machines» 5.

A errônea concepção da ciência 6 e de seus objetivos é responsável pelo dualismo divisionista entre corpo e mente, entre a medicina científica e a prática médica do dia-a-dia. Historicamente a medicina foi a primeira ciência humana, cuando o homem procurou compreender as situações que ocasionavam dor e sofrimento, buscou seu alívio, assim como a conservação e o prolongamento de sua vida 3 . As questões humanistas, ao contrário de alheias à ciência médica, constituem o seu próprio núcleo, em torno do qual toda física e química devem se dispor. É papel, portanto, das entidades responsáveis pela educação médica, propiciar oportunidades de desenvolvimento de traços e virtudes humanísticas na prática médica, sem os quais a medicina foge às suas finalidades.

A ABN, em seus programas educacionais, deve enfatizar as qualidades humanísticas do neurologista, na relação médico-paciente e em suas interações com os colegas e a sociedade. Em suas relações com o paciente, o neurologista deve comportar-se com integridade, respeito e compaixão ${ }^{10}$. A «integridade» refere-se à sua honestidade na avaliação de sua própria competência e habilidades. O «respeito», a seu compromisso de honrar os direitos e desejos do paciente em relação a si próprio e à sua doença. A «compaixão» refere-se à sua sensibilidade para confortar e ajudar os pacientes, pela percepção das necessidades especiais trazidas pela doença e sofrimento. Estas qualidades fundamentais são melhor cultivadas e desenvolvidas em meio apropriado e pelo contacto direto com os professores, durante o curso de graduação e, na residência médica, pela vivência das situações de fato 7 . No entanto, as dimensões cognitivas da ética e da moral podem ser discutidas e ensinadas fora das enfermarias e ambulatórios 9 .

Temas humanísticos e de natureza ética e moral, com que se deparam os neurologistas em sua vida diária devem ser incluídos nos programas curriculares da residência médica em neurologia. Além disso, a ABN deve promover apreciações e debates sobre esses temas em simpósios específicos, inclui-los em seus Congressos assim como incentivar sua publicação nos «Arquivos de Neuro-Psiquiatria».

A ABN não desempenhará completamente seu papel no ensino da neurologia enquanto não abordar a dimensionalização humanística do neurologista como questão fundamental no direcionamento de sua formação científica e no balisamento de seu comportamento médico.

\section{REFERÊNCIAS}

1. Badin A. Relatório das Atividades 1982-1984, Comissão de Ensino, Academia Brasileira de Neurologia, 1984

2. Cavalcanti JL. Relatório das Atividades 1986-1988, Comissão de Ensino, Academia Brasileira de Neurologia, 1988.

3. Engel G. Physician-scientists and scientific physicians: resolving the humanism-science dichotomy. Am J Med 1987, 82 :107.

4. Estatuto. Academia Brasileira de Neurologia. Arq Neuro-Psiquiat (São Paulo) 1987, 45 (Apêndice) : 9.

5. Gull WW. Clinical Observation in Relation to Medicine in Modern Times. In Acland DT (ed): A Collection of the Published Writings of William Witley Gull, Bart., M.D.F.R.S. Memoirs and Addresses. London: New Syndenham Society, 1898.

6. Harrisos AJ. Common elements and interconnections. Science 1984, 224-939.

7. Lana MA. A residência médica e o título de especialista em neurologia. Arq Neuro-ᄀ -Psiquiat (São Paulo) 1989, 47:503.

8. Lana MA. Relatório das Atividades 1988-1990, Comissão de Ensino, Academia Brasileira de Neurologia, 1990

9. Subcommittee on Evaluation of Humanistic Qualities in the Internist, American Board of Internal Medicine. Evaluation of humanistic qualities in the internist. Ann Inter Med $1963,99: 720$.

10. Wittig E. Relatório das Atividades 1984-1986, Comissão de Ensino, Academia Brasileira de Neurologia, 1986. 